\title{
Prevalence of lifetime DSM-IV affective disorders among older African Americans, Black Caribbeans, Latinos, Asians and Non-Hispanic White people
}

\author{
Amanda Toler Woodward ${ }^{1}$, Robert Joseph Taylor ${ }^{2}$, Kai McKeever Bullard ${ }^{4}$, Maria P. Aranda ${ }^{5}$, Karen D. Lincoln ${ }^{5}$ \\ and Linda M. Chatters ${ }^{2,3}$ \\ ${ }^{1}$ School of Social Work, Michigan State University, East Lansing, MI, USA \\ ${ }^{2}$ School of Social Work, Institute for Social Research, University of Michigan, Ann Arbor, MI, USA \\ ${ }^{3}$ School of Public Health, University of Michigan, Ann Arbor, MI, USA \\ ${ }^{4}$ Northrop Grumman Corporation, Atlanta, GA, USA \\ ${ }^{5}$ School of Social Work, University of Southern California, Los Angeles, CA, USA \\ Correspondence to: A. Woodward, PhD, E-mail: awoodwar@msu.edu
}

\begin{abstract}
Objectives: The purpose of this study is to estimate lifetime prevalence of seven psychiatric affective disorders for older non-Hispanic White people, African Americans, Caribbean Black people, Latinos, and Asian Americans and examine demographic, socioeconomic, and immigration correlates of those disorders.

Design: Data are taken from the older sub-sample of the Collaborative Psychiatric Epidemiology Surveys. Selected measures of lifetime DSM-IV psychiatric disorders were examined (i.e., panic disorder, agoraphobia, social phobia, generalized anxiety disorder, post-traumatic stress disorder, major depressive disorder, and dysthymia).

Setting: Community epidemiologic survey.

Participants: Nationally representative sample of adults 55 years and older $(n=3046)$.

Measurements: Disorders were assessed using the DSM-IV World Mental Health Composite International Diagnostic Interview.

Results: Major depressive disorder and social phobia were the two most prevalent disorders among the seven psychiatric conditions. Overall, non-Hispanic White people and Latinos consistently had higher prevalence rates of disorders, African Americans had lower prevalence of major depression and dysthymia, and Asian Americans were typically less likely to report affective disorders than those of their counterparts. There is variation across groups in the association of demographic, socioeconomic, and immigration variables with disorders.

Conclusions: This study furthers our understanding of the racial and ethnic differences in the prevalence of DSM-IV disorders among older adults and the correlates of those disorders. It highlights the importance of examining both between-group and within-group differences in disorders and the complexity of the mechanisms associated with differences across groups. Findings from this study underscore the need for future research that more clearly delineates subgroup differences and similarities. Copyright (C) 2011 John Wiley \& Sons, Ltd.
\end{abstract}

Key words: depression; anxiety; older; race; ethnicity

History: Received 3 December 2010; Accepted 27 July 2011; Published online 10 October 2011 in Wiley Online Library

(wileyonlinelibrary.com).

DOI: 10.1002 /gps.2790

\section{Objective}

Previous epidemiological studies have provided insight into symptoms of depression and anxiety among older adults through symptom checklist measures. These studies find that symptoms decline over time although older adults experience significant impairment (Ramsawh et al., 2009), and symptoms of depression and anxiety 
increase again after the mid-1970s (Teachman, 2006). Depressive and anxiety symptoms are associated with increased disability particularly among older adults (Brenes et al., 2008), and persistent depressive symptoms are associated with cognitive deficits in older adults over time (Mojtabai and Olfson, 2004). Furthermore, White people have higher levels of symptoms than other groups, both across age groups and among older adults (Cohen et al., 2006; Mehta et al., 2003).

Large nationally representative datasets build on this research by examining the full range of diagnostic categories and including older adults who meet criteria for disorders but have not used mental health services or received mental health treatment. Similar to symptom-based research, studies of this type have found that the prevalence of depressive and anxiety disorders declines with age (Scott et al., 2008; Hasin et al., 2005; Grant et al., 2005; Mojtabai and Olfson, 2004) even when controlling for comorbid physical illness or pain (Scott et al., 2008). In fact, adults 65 years and older have the lowest prevalence of disorders including major depression (Hasin et al., 2005; Mojtabai and Olfson, 2004), and generalized anxiety (Grant et al., 2005). Psychiatric disorders are associated with functional decline (Callahan et al., 2005), and poor physical functioning and illness (Cohen et al., 2006; Lyness et al., 2006). As observed in studies focusing on symptoms, White people have higher prevalence of mood and anxiety disorders compared with other racial/ethnic groups. However, the variability in prevalence across specific disorders and racial/ ethnic groups is complex (Smith et al., 2006; Hasin et al., 2005; Grant et al., 2005) with the lower lifetime risk for ethnic groups compared with White people stemming from a small subset of disorders and beginning early in life (Breslau et al., 2006).

This study builds on this body of research as well as recent studies using data from the Collaborative Psychiatric Epidemiology Surveys (CPES) (Ford et al., 2007; Jimenez et al., 2010; González et al., 2010) to investigate differences in the prevalence of DSM-IV disorders in a national sample of five racial/ ethnic groups 55 years and older.

Using data from the NSAL, a subset of the CPES, Ford et al. (2007) found that 23\% of African Americans 55 years and older met diagnostic criteria for one lifetime disorder, and 8.5\% met criteria for a 12-month disorder. Alcohol abuse, PTSD, and major depression were most prevalent, and those older adults 55-64 years had the highest prevalence whereas those 75 years and older the lowest. The present study extends this work to include both between-group and withingroup analysis of older non-Hispanic White people,
African Americans, Black Caribbeans, Asian Americans, and Latinos.

Consistent with previous studies, Jimenez et al. (2010) found that White people 60 years and older in the CPES data had significantly higher prevalence for several lifetime disorders compared with Asian Americans, African Americans, and Black Caribbeans. White people did not differ significantly from Latinos, however, in terms of lifetime prevalence, and there were no differences across racial/ethnic groups in the prevalence of 12-month disorders. The work of Jimenez et al. (2010) also enhances our understanding of the relationship between immigration and diagnosis among older adults. In particular, their finding that the prevalence of disorders did not differ between older adults born in the USA and immigrants is contrary to what has been reported in previous studies (Ortega et al., 2000; Alegría et al., 2008). Similarly, González et al. (2010) found that the prevalence of major depressive disorder was greater for US versus foreign-born respondents, except for foreign-born respondents 65 years and older. Neither Jimenez et al. nor González et al. examine demographic and socioeconomic correlates. The present study builds on their work by examining the prevalence of selected psychiatric disorders in conjunction with demographic, socioeconomic, and immigration factors, both across and within five racial/ethnic groups of older adults.

\section{Methods}

\section{Sample}

This study utilizes data from three surveys that comprise the CPES, which include data about the prevalence of mental disorders, their associated impairments, and their treatment in a representative sample of the adult population in the USA. Non-Hispanic White people in our study are from the National Comorbidity Survey Replication (NCS-R), African Americans and Black Caribbeans are from the National Survey of American Life (NSAL), and Latinos and Asian Americans are from the National Latino and Asian American Study (NLAAS). Data for each survey were collected in faceto-face interviews by the Institute for Social Research at the University of Michigan between February 2001 and November 2003. The CPES surveys share the multistage area probability sample designs common to the national surveys conducted by the Survey Research Center (Heeringa et al., 2004; Pennell et al., 2004). In addition, the surveys share a common set of objectives and instrumentation and were designed to allow 
integration of design-based analysis weights to combine data sets as though they were a single, nationally representative study (Heeringa \& Berglund, 2007). Final response rates for the CPES surveys two-phase sample designs were computed using the American Association of Public Opinion Research (AAPOR) guidelines for Response Rate 3 (American Association for Public Opinion Research, 2006) and range from 70.9\% for the NCS-R to $73.2 \%$ for the NLAAS. (See Heeringa et al., 2004 for a more detailed discussion of the CPES samples). The analytic samples for this study are adults from the CPES who are 55 years and over $(n=3046)$.

\section{Measures}

Diagnostic assessment. The CPES surveys used the World Mental Health Composite International Diagnostic Interview (WMH-CIDI), a fully structured diagnostic interview used to assess mental disorders. The WMH-CIDI is an expanded version of the World Health Organization CIDI that includes expanded diagnostic sections as well as a greater number of disorders (Kessler and Üstün, 2004). The mental disorders examined in the present analysis include anxiety disorders (panic disorder, agoraphobia, social phobia, generalized anxiety disorder, posttraumatic stress disorder) and mood disorders (major depressive disorder, dysthymia). Bipolar disorder was excluded because it was not assessed in the NLAAS.

Covariates. The main predictor of interest is race/ethnicity (non-Hispanic White, African American, Black Caribbean, Asian, or Latino) determined by self-report at the time of household screening. Covariates include age, gender, and education (fewer than 12 years, 12 years, or 13 years or more of education). Household income was measured by a three-category variable, which indexes income to the poverty line based on 2001 Census data. Measures of employment status (employed, not employed) and marital status (married, not married) are dichotomized whereas region is measured by a four-category variable (Northeast, Midwest, South, or West).

Analysis strategy. Cross-tabulations illustrate differences across racial and ethnic groups in demographic, socioeconomic, and immigration factors as well as prevalence of disorders. Percentages are weighted based on the distribution of the racial and ethnic groups in the population. The standard errors reflect the recalculation of variance using the study's complex design.
Design-corrected F statistics were used to test differences between race/ethnic groups. Predicted prevalence estimates of affective disorder were calculated for each racial/ethnic group, adjusting for age, gender, employment status, poverty-income level, education, marital status, region, and nativity. Multivariate logistic regression models predict the association of covariates with the odds of having a mood or anxiety disorder. All analyses were conducted with SUDAAN 10.0 using the Taylor linearization method for calculating the complex design-based estimates of variance. A $p$-value $<0.05$ was considered statistically significant.

\section{Results}

Demographic characteristics of the sample are presented in Table 1. A higher proportion of Caribbean Black people are in the youngest age group (52.1\%) compared with the other racial and ethnic groups, whereas a higher proportion of non-Hispanic White people are 75 years or older $(28.3 \%)$. Consistent with most samples of older adults, there are slightly more women than men with the exception of Caribbean Black people, the youngest of the five groups, for whom the percentage of men and women is equal. Asian Americans have the highest level of education (51.8\% with $\geq 13$ years of education), whereas Latinos have the lowest level of education $(64.5 \%$ with $<12$ years). Consistent with their younger ages, more than half of Caribbean Black people were employed at the time of the interview (58.7\%). A higher proportion of Latinos (18.8\%) and Asians $(17.7 \%)$ have incomes below the poverty level compared with the other groups. More African Americans (55.9\%) and Caribbean Black people (47.9\%) have incomes 100 to $300 \%$ of poverty level whereas a higher proportion of White people $(63.7 \%)$ have incomes at or greater than $300 \%$ of poverty level. More Asian Americans are married (76.8\%) followed by non-Hispanic White people (63.2\%). African Americans have the lowest proportion of married persons (40.4\%). A higher proportion of Caribbean Black people $(48.5 \%)$ live in the Northeastern USA whereas more African Americans (56\%) live in the South. More Asians (74.8\%) live in the Western USA. White people and African Americans are almost entirely US-born whereas $28.3 \%$ of Caribbean Black people, $36.7 \%$ of Latinos, and $21.1 \%$ of Asians were born in the USA. Among those who were foreign-born, most have lived in the USA for 20 years or more, with a higher proportion of Asians having lived in the USA for less than 20 years. More Latinos (60.5\%) were less than 
Table 1 Characteristics of 3046 adults 55 years and older by race/ethnicity from the Collaborative Psychiatric Epidemiology Surveys, 2001-3003

\begin{tabular}{|c|c|c|c|c|c|c|c|c|c|c|c|}
\hline & \multirow{2}{*}{\multicolumn{2}{|c|}{$\begin{array}{c}\begin{array}{c}\text { Non-Hispanic } \\
\text { White }\end{array} \\
n=1130\end{array}$}} & \multirow{2}{*}{\multicolumn{2}{|c|}{$\begin{array}{c}\begin{array}{c}\text { African } \\
\text { American }\end{array} \\
n=780\end{array}$}} & \multirow{2}{*}{\multicolumn{2}{|c|}{$\begin{array}{c}\begin{array}{c}\text { Caribbean } \\
\text { Black }\end{array} \\
n=262\end{array}$}} & \multirow{2}{*}{\multicolumn{2}{|c|}{$\begin{array}{c}\begin{array}{c}\text { Latino } \\
\text { American }\end{array} \\
\qquad n=498\end{array}$}} & \multirow{2}{*}{\multicolumn{2}{|c|}{$\begin{array}{c}\begin{array}{c}\text { Asian } \\
\text { American }\end{array} \\
n=376\end{array}$}} & \multirow[b]{3}{*}{$p$-value ${ }^{b}$} \\
\hline & & & & & & & & & & & \\
\hline & $\%^{a}$ & SE & $\%$ & SE & $\%$ & SE & $\%$ & SE & $\%$ & SE & \\
\hline \multicolumn{12}{|l|}{ Age group, \% } \\
\hline $55-64$ years & 40.0 & 2.5 & 47.4 & 2.1 & 52.1 & 5.6 & 46.0 & 3.6 & 46.7 & 3.7 & $<0.001$ \\
\hline $65-74$ years & 31.7 & 2.3 & 36.7 & 2.3 & 39.8 & 5.3 & 35.7 & 3.4 & 36.3 & 3.7 & 0.462 \\
\hline $75+$ years & 28.3 & 1.9 & 16.0 & 1.6 & 8.2 & 2.1 & 18.3 & 2.3 & 17.1 & 1.7 & $<0.001$ \\
\hline Female, \% & 56.4 & 2.1 & 59.3 & 1.8 & 50.4 & 5.4 & 56.0 & 3.1 & 54.8 & 2.6 & $<0.001$ \\
\hline \multicolumn{12}{|l|}{ Education, \% } \\
\hline$<12$ years & 22.1 & 2.2 & 38.4 & 2.2 & 36.1 & 5.5 & 64.5 & 3.2 & 28.5 & 3.0 & $<0.001$ \\
\hline 12 years & 35.1 & 2.2 & 31.8 & 2.0 & 31.3 & 3.7 & 15.8 & 2.1 & 19.8 & 2.5 & $<0.001$ \\
\hline$\geq 13$ years & 42.8 & 2.4 & 29.8 & 2.4 & 32.6 & 5.6 & 19.7 & 2.4 & 51.8 & 3.4 & $<0.001$ \\
\hline Employed, \% & 34.2 & 1.5 & 33.9 & 2.7 & 58.7 & 4.2 & 27.6 & 3.6 & 39.8 & 4.1 & $<0.001$ \\
\hline \multicolumn{12}{|l|}{ Poverty-income level, \% } \\
\hline$<100 \%$ poverty & 3.9 & 0.7 & 5.9 & 1.1 & 3.0 & 0.9 & 18.8 & 2.2 & 17.7 & 2.4 & \\
\hline $100-300 \%$ poverty & 32.4 & 1.9 & 55.9 & 2.6 & 47.9 & 3.9 & 41.4 & 3.6 & 27.6 & 2.6 & \\
\hline$\geq 300 \%$ poverty & 63.7 & 1.8 & 38.2 & 2.5 & 49.1 & 3.9 & 39.9 & 3.4 & 54.7 & 3.6 & $<0.001$ \\
\hline Married/partner, \% & 63.2 & 1.8 & 40.4 & 2.3 & 54.6 & 7.0 & 57.8 & 2.9 & 76.8 & 2.8 & $<0.001$ \\
\hline \multicolumn{12}{|l|}{ Region, \% } \\
\hline Northeast & 22.2 & 3.9 & 15.7 & 2.1 & 48.5 & 8.3 & 20.6 & 2.8 & 13.8 & 7.2 & \\
\hline Midwest & 28.3 & 3.0 & 19.3 & 2.8 & 8.0 & 2.2 & 7.6 & 2.5 & 3.5 & 2.0 & \\
\hline South & 30.9 & 3.8 & 56.0 & 3.2 & 36.4 & 9.8 & 38.6 & 5.4 & 8.0 & 2.2 & \\
\hline West & 18.6 & 3.0 & 9.0 & 2.3 & 7.1 & 3.4 & 33.2 & 4.9 & 74.8 & 7.3 & $<0.001$ \\
\hline \multirow{2}{*}{\multicolumn{12}{|c|}{ Among foreign-born only: }} \\
\hline & & & & & & & & & & & \\
\hline \multicolumn{12}{|l|}{ Years in the USA, \% } \\
\hline$<10$ years & \multicolumn{2}{|c|}{$\mathrm{n} / \mathrm{a}$} & \multicolumn{2}{|c|}{$\mathrm{n} / \mathrm{a}$} & 7.8 & 2.9 & 9.6 & 2.0 & 21.2 & 2.5 & \\
\hline $11-<20$ years & \multicolumn{2}{|c|}{$\mathrm{n} / \mathrm{a}$} & \multicolumn{2}{|c|}{$\mathrm{n} / \mathrm{a}$} & 17.6 & 3.0 & 8.5 & 1.8 & 25.8 & 2.9 & \\
\hline$\geq 20$ years & \multicolumn{2}{|c|}{$\mathrm{n} / \mathrm{a}$} & \multicolumn{2}{|c|}{$\mathrm{n} / \mathrm{a}$} & 74.3 & 4.6 & 80.6 & 2.7 & 53.1 & 4.4 & \\
\hline Age $<35$ years at immigration, $\%$ & \multicolumn{2}{|c|}{$\mathrm{n} / \mathrm{a}$} & \multicolumn{2}{|c|}{$\mathrm{n} / \mathrm{a}$} & 42.8 & 4.5 & 60.5 & 4.1 & 30.5 & 4.8 & $<0.001$ \\
\hline
\end{tabular}

${ }^{a}$ Weighted percentage and standard error (SE) adjusted for complex survey design. Percentages may not total 100 because of rounding.

${ }^{\mathrm{b}}$ Adjusted Wald $F$-Test.

35 years old at immigration compared with Caribbean Black people (42.8\%) and Asians (30.5\%).

Table 2 presents the predicted prevalence of lifetime DSM-IV affective disorders by race and ethnicity adjusting for age, gender, employment status, povertyincome level, education, marital status, region, and nativity. Overall, non-Hispanic White people $(23.5 \%)$ and Latinos $(22.8 \%)$ have the highest prevalence of any affective disorder, whereas Asian Americans (12.3\%) and African Americans (13.7\%) have the lowest prevalence, and Caribbean Black people fall in between $(17.5 \%)$. This pattern is true for one affective disorder, or two or more disorders.

Non-Hispanic White people have the highest prevalence of any anxiety disorder $(16.8 \%)$ followed by Latinos (15.2\%). A smaller proportion of Asian Americans have any anxiety disorder $(7.9 \%)$ whereas African Americans and Caribbean Black people have similar prevalence ( 11.0 vs $11.5 \%$, respectively). NonHispanic White people and Latinos also have the highest prevalence for social phobia and generalized anxiety disorder.
With regard to mood disorders, Latinos (13.9\%) and non-Hispanic White people $(13.8 \%)$ had the highest prevalence of any mood disorder followed by Caribbean Black people (11.2\%). African Americans (5.4\%) and Asian Americans (6.4\%) had the lowest prevalence. This pattern is largely caused by differences in the prevalence of major depressive disorder.

Table 3 presents odds ratios and 95\% confidence intervals for logistic regression models of any lifetime mood or anxiety disorder. Results are presented with both non-Hispanic White people and African Americans (the two largest groups) as the reference group for race/ethnicity. Compared with African Americans, White people and Latinos were more likely to meet criteria for a mood or anxiety disorder. No other groups differed significantly compared with White people. Older age cohorts were less likely to meet criteria for either a mood or anxiety disorder, and men were less likely than women to have a disorder. Respondents who were not working were more likely to have a disorder, and those who were not married were more likely to have a disorder compared 


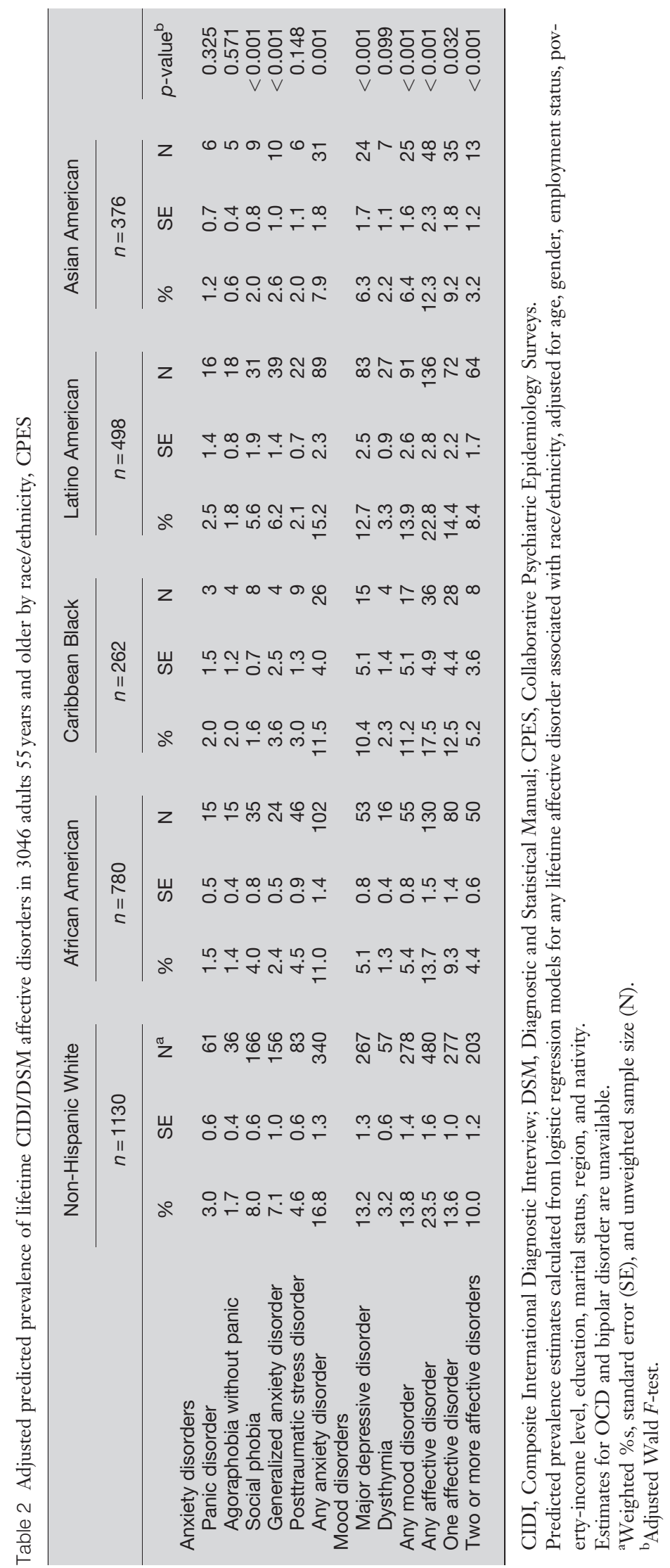


Table 3 Multivariate-adjusted odds ratios for any lifetime CIDI/DSM mood or anxiety disorder associated with selected demographics

\begin{tabular}{|c|c|c|c|c|}
\hline & \multicolumn{2}{|c|}{ Mood disorders } & \multicolumn{2}{|c|}{ Anxiety disorders } \\
\hline & OR & $95 \% \mathrm{Cl}$ & OR & $95 \% \mathrm{Cl}$ \\
\hline \multicolumn{5}{|l|}{ Race/ethnicity } \\
\hline African American as reference & 1.00 & & 1.00 & \\
\hline White & 2.99 & $2.07-4.31$ & 1.78 & $1.27-2.49$ \\
\hline Caribbean Black & 3.17 & $1.00-10.08$ & 1.70 & $0.71-4.07$ \\
\hline Latino & 3.91 & $2.11-7.24$ & 2.21 & $1.29-3.79$ \\
\hline Asian & 1.61 & $0.78-3.33$ & 1.09 & $0.53-2.22$ \\
\hline$p$-value ${ }^{a}$ & $<0.001$ & & 0.001 & \\
\hline \multicolumn{5}{|l|}{ White as reference } \\
\hline African American & 0.33 & $0.23-0.48$ & 0.56 & $0.40-0.79$ \\
\hline Caribbean Black & 1.06 & $0.34-3.28$ & 0.95 & $0.41-2.24$ \\
\hline Latino & 1.31 & $0.74-2.32$ & 1.24 & $0.75-2.07$ \\
\hline Asian & 0.54 & $0.28-1.02$ & 0.61 & $0.32-1.18$ \\
\hline$p$-value & $<0.001$ & & 0.001 & \\
\hline \multicolumn{5}{|l|}{ Age } \\
\hline $55-64$ & 1.00 & & 1.00 & \\
\hline $65-74$ & 0.57 & $0.40-0.83$ & 0.48 & $0.36-0.64$ \\
\hline $75+$ & 0.24 & $0.15-0.36$ & 0.22 & $0.13-0.38$ \\
\hline$p$-value & $<0.001$ & & $<0.001$ & \\
\hline \multicolumn{5}{|l|}{ Gender } \\
\hline Female & 1.00 & & 1.00 & \\
\hline Male & 0.50 & $0.37-0.69$ & 0.68 & $0.51-0.91$ \\
\hline$p$-value & $<0.001$ & & 0.009 & \\
\hline \multicolumn{5}{|l|}{ Education } \\
\hline$<$ High school & 0.99 & $0.66-1.49$ & 0.97 & $0.67-1.41$ \\
\hline High school & 0.80 & $0.56-1.13$ & 0.69 & $0.50-0.97$ \\
\hline$>$ High school & 1.00 & & 1.00 & \\
\hline$p$-value & 0.34 & & 0.08 & \\
\hline \multicolumn{5}{|l|}{ Employment status } \\
\hline Working & 1.00 & & 1.00 & \\
\hline Not working & 1.36 & $1.04-1.79$ & 1.47 & $1.07-2.01$ \\
\hline$p$-value & 0.03 & & 0.02 & \\
\hline \multicolumn{5}{|l|}{ Poverty income level } \\
\hline$<100 \%$ poverty & 1.34 & $0.76-2.36$ & 1.62 & $0.98-2.66$ \\
\hline $100-300 \%$ poverty & 1.00 & & 1.00 & \\
\hline$\geq 300 \%$ poverty & 1.08 & $0.76-1.55$ & 0.99 & $0.73-1.34$ \\
\hline$p$-value & 0.59 & & 0.16 & \\
\hline \multicolumn{5}{|l|}{ Marital status } \\
\hline Married & 1.00 & & 1.00 & \\
\hline Not married & 1.77 & $1.38-2.46$ & 1.69 & $1.17-2.46$ \\
\hline$p$-value & $<0.001$ & & 0.01 & \\
\hline \multicolumn{5}{|l|}{ Region } \\
\hline Northeast & 1.3 & $0.73-2.31$ & 1.28 & $0.80-2.04$ \\
\hline Midwest & 0.96 & $0.60-1.54$ & 1.27 & $0.83-1.93$ \\
\hline South & 1.00 & & 1.00 & \\
\hline West & 1.37 & $0.89-2.10$ & 1.37 & $0.90-2.09$ \\
\hline$p$-value & 0.34 & & 0.46 & \\
\hline \multicolumn{5}{|l|}{ Nativity } \\
\hline US-born & 1.00 & & 1.00 & \\
\hline Foreign-born & 0.54 & $0.33-0.90$ & 0.47 & $0.31-0.71$ \\
\hline$p$-value & 0.02 & & $<0.001$ & \\
\hline
\end{tabular}

CIDI, Composite International Diagnostic Interview; DSM, Diagnostic and Statistical Manual; OR, odds ratio; CI, confidence interval.

Data presented are odds ratios and $95 \%$ confidence intervals estimated from multivariate logistic regression models of any lifetime mood disorder or any lifetime anxiety disorder and selected characteristics.

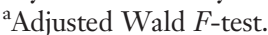

with those who were married. Respondents who were born outside the USA were less likely to have a disorder than US-born older adults.

Tables 4 and 5 present within group, differences in prevalence for any mood disorder and any anxiety disorder, respectively. Because of the small number of cases, these tables show bivariate comparisons only. The prevalence of mood disorders declines with age among White people and African Americans whereas anxiety disorders decline with age among White 
Table 4 Prevalence of lifetime CIDI/DSM mood disorder by selected sociodemographic characteristics and ethnicity, CPES

\begin{tabular}{|c|c|c|c|c|c|c|c|c|c|c|c|c|c|c|c|}
\hline & \multicolumn{3}{|c|}{ White } & \multicolumn{3}{|c|}{ African American } & \multicolumn{3}{|c|}{ Black Caribbean } & \multicolumn{3}{|c|}{ Latino } & \multicolumn{3}{|c|}{ Asian } \\
\hline & $\%$ & SE & $\mathrm{N}$ & $\%$ & SE & $\mathrm{N}$ & $\%$ & SE & $\mathrm{N}$ & $\%$ & SE & $\mathrm{N}$ & $\%$ & SE & $\mathrm{N}$ \\
\hline \multicolumn{16}{|l|}{ Age } \\
\hline $55-64$ & 18.6 & 1.9 & 149 & 8.9 & 1.7 & 36 & 18.4 & 8.7 & 8 & 17.4 & 3.7 & 51 & 5.3 & 1.8 & 13 \\
\hline $65-74$ & 12.9 & 1.8 & 95 & 5.9 & 1.5 & 16 & 5.6 & 4 & 5 & 15.7 & 3.3 & 25 & 7.3 & 3.6 & 7 \\
\hline $75+$ & 6.8 & 1.6 & 34 & 1.7 & 0.8 & 3 & 7.8 & 4.2 & 4 & 12.9 & 4.1 & 15 & 8.9 & 4.2 & 5 \\
\hline $\mathrm{F}_{2}^{\mathrm{a}}$ & $15.6^{\star \star \star}$ & & & $7.6^{\star \star \star}$ & & & 0.8 & & & 0.3 & & & 0.4 & & \\
\hline \multicolumn{16}{|l|}{ Gender } \\
\hline Male & 8.3 & 1.4 & 68 & 5.8 & 1.5 & 16 & 21.5 & 11.8 & 7 & 9.3 & 2.6 & 24 & 5.3 & 2.1 & 11 \\
\hline Female & 17.5 & 1.6 & 210 & 7.2 & 1.1 & 39 & 3.4 & 1.3 & 10 & 21.2 & 3.6 & 67 & 7.7 & 2.6 & 14 \\
\hline \multicolumn{16}{|l|}{ Employment status } \\
\hline Working & 14.4 & 1.6 & 107 & 7.1 & 1.4 & 20 & 11.7 & 7.7 & 7 & 6.4 & 2.3 & 13 & 6.3 & 2.6 & 9 \\
\hline Not working & 12.9 & 1.5 & 170 & 6.4 & 1.2 & 35 & 13.4 & 9 & 10 & 19.6 & 3 & 78 & 6.8 & 1.9 & 16 \\
\hline $\begin{array}{l}\mathrm{F}_{1} \\
\text { Education }\end{array}$ & 0.9 & & & 0.2 & & & 0 & & & $14.7^{\star \star \star}$ & & & 0 & & \\
\hline$<12$ years & 13.2 & 2.7 & 52 & 6.9 & 1.1 & 25 & 14.2 & 10.7 & 6 & 16.7 & 3.2 & 62 & 2.8 & 1.4 & 5 \\
\hline 12 years & 11.9 & 1.5 & 84 & 8.9 & 2.2 & 21 & 2.1 & 1.5 & 3 & 20.7 & 5.1 & 17 & 8.8 & 5.3 & 5 \\
\hline$>12$ years & 14.9 & 1.5 & 142 & 4 & 1.2 & 9 & 20.3 & 12.4 & 8 & 9.9 & 3.7 & 12 & 7.9 & 2.3 & 15 \\
\hline $\begin{array}{l}\mathrm{F}_{2} \\
\text { Poverty-income level }\end{array}$ & 1.5 & & & 2.1 & & & \multicolumn{4}{|c|}{ Poverty-income level } & & & 2.4 & & \\
\hline$<100 \%$ poverty & 21.4 & 6.2 & 13 & 11.7 & 4.9 & 8 & 15.1 & 8.3 & 3 & 15.6 & 5.6 & 17 & 15.9 & 6.3 & 8 \\
\hline $100-300 \%$ poverty & 12.9 & 1.7 & 88 & 7.6 & 1.3 & 36 & 9.6 & 7.8 & 7 & 15.3 & 2.7 & 44 & 7.2 & 3.9 & 7 \\
\hline$\geq 300 \%$ poverty & 13.3 & 1.4 & 177 & 3.6 & 0.9 & 12 & 13.1 & 9.2 & 7 & 16.9 & 4.3 & 30 & 3.3 & 1 & 10 \\
\hline $\begin{array}{l}\mathrm{F}_{2} \\
\text { Marital status }\end{array}$ & \multicolumn{10}{|c|}{ Marital status } & & & 2 & & \\
\hline Married & 11 & 1.3 & 138 & 5.5 & 1.5 & 15 & 17.9 & 8.5 & 7 & 12.9 & 2.4 & 43 & 5.3 & 2 & 13 \\
\hline Not married & 17.6 & 2.4 & 140 & 7.4 & 1.2 & 40 & 5.8 & 2.9 & 10 & 20.2 & 3.9 & 48 & 10.9 & 3.5 & 12 \\
\hline $\mathrm{F}_{1}$ & $6.9^{\star \star}$ & & & 0.9 & & & 2.2 & & & $4.6^{\star}$ & & & 1.4 & & \\
\hline \multicolumn{16}{|l|}{ Region } \\
\hline Northeast & 15.1 & 4 & 54 & 11.5 & 1.5 & 14 & 13 & 7.5 & 13 & 22.6 & 5.3 & 27 & 4.5 & 4.7 & 1 \\
\hline Midwest & 10.9 & 1.7 & 76 & 7.5 & 2.7 & 11 & 0 & 0 & 0 & 11.2 & 5.8 & 5 & 22.2 & 11.1 & 1 \\
\hline South & 12.7 & 2.1 & 81 & 5.4 & 0.9 & 31 & 1.3 & 1.1 & 3 & 14.9 & 3.7 & 47 & 4.3 & 4.3 & 1 \\
\hline West & 16.6 & 2.5 & 67 & 0 & 0 & 0 & 66.1 & 35.1 & 1 & 14.2 & 5.8 & 12 & 6.5 & 1.5 & 22 \\
\hline $\mathrm{F}_{3}$ & 1.3 & & & $n / a$ & & & $\mathrm{n} / \mathrm{a}$ & & & 0.8 & & & 0.4 & & \\
\hline \multicolumn{16}{|l|}{ Nativity } \\
\hline US-born & 13.9 & 1.4 & 270 & 6.4 & 0.9 & 55 & 27 & 12.8 & 4 & 12 & 4.9 & 12 & 8 & 3.3 & 6 \\
\hline Foreign-born & 5.7 & 2.3 & 8 & 2.2 & 2.3 & 1 & 5.4 & 2.2 & 13 & 18.3 & 2.2 & 79 & 6.2 & 1.9 & 19 \\
\hline $\mathrm{F}_{1}$ & $5.3^{*}$ & & & 1.7 & & & 1.8 & & & 1.8 & & & 0.2 & & \\
\hline \multicolumn{16}{|c|}{$\begin{array}{l}\text { Among foreign-born only: } \\
\text { Years in the USA }\end{array}$} \\
\hline$<10$ years & & & & & & & 33.9 & 17.1 & 3 & 22.6 & 8.6 & 11 & 12.2 & 62 & 6 \\
\hline $11-<20$ years & & & & & & & 1.4 & 1 & 2 & 7.5 & 3.4 & 6 & 9 & 5.4 & 7 \\
\hline$\geq 20$ years & & & & & & & 3.3 & 1.4 & 8 & 19.2 & 2.3 & 62 & 2.5 & 1.2 & 6 \\
\hline $\mathrm{F}_{2}$ & & & & & & & 1.2 & & & 2 & & & 1.6 & & \\
\hline \multicolumn{16}{|l|}{ Age at immigration } \\
\hline $\begin{array}{l}<35 \text { years } \\
\geq 35 \text { years }\end{array}$ & & & & & & & $\begin{array}{l}3.2 \\
7.1\end{array}$ & $\begin{array}{l}2 \\
3.4\end{array}$ & $\begin{array}{l}3 \\
9\end{array}$ & $\begin{array}{l}16.5 \\
21.8\end{array}$ & $\begin{array}{l}2.7 \\
4.8\end{array}$ & $\begin{array}{l}31 \\
42\end{array}$ & $\begin{array}{l}2.1 \\
8\end{array}$ & $\begin{array}{l}1.3 \\
2.8\end{array}$ & $\begin{array}{r}4 \\
15\end{array}$ \\
\hline $\mathrm{F}_{1}$ & & & & & & & 1.1 & & & 0.9 & & & 2.9 & & \\
\hline
\end{tabular}

CIDI, Composite International Diagnostic Interview; DSM, Diagnostic and Statistical Manual; CPES, Collaborative Psychiatric Epidemiology Surveys; SE, Standard error.

Any mood disorder includes dysthymia and major depression.

Prevalence estimates calculated from cross-tabulation of any lifetime mood disorder and selected characteristic stratified by race/ethnicity.

a Test of association determined by adjusted Wald $F$-test for each race/ethnicity.

${ }^{*} p<0.05$.

${ }^{* *} p<0.01$.

${ }^{* * *} p<0.001$.

people only. Compared with men, significantly higher proportions of White people and Latino women have a mood disorder, and more White people and African American women meet criteria for anxiety disorders.
Significantly more Latinos who are not working (19.6\%) suffer from mood disorders than those who are working $(6.4 \%)$. For anxiety disorders, significantly more Asians who are not working (12\%) meet criteria 
Table 5 Prevalence of lifetime CIDI/DSM anxiety disorders by selected sociodemographic characteristics and ethnicity, CPES

\begin{tabular}{|c|c|c|c|c|c|c|c|c|c|c|c|c|c|c|c|}
\hline & \multicolumn{3}{|c|}{ White } & \multicolumn{3}{|c|}{ African American } & \multicolumn{3}{|c|}{ Black Caribbean } & \multicolumn{3}{|c|}{ Latino } & \multicolumn{3}{|c|}{ Asian } \\
\hline & $\%$ & SE & $\mathrm{N}$ & $\%$ & SE & $\mathrm{N}$ & $\%$ & SE & $\mathrm{N}$ & $\%$ & SE & $\mathrm{N}$ & $\%$ & SE & $\mathrm{N}$ \\
\hline \multicolumn{16}{|l|}{ Age } \\
\hline $55-64$ & 23.6 & 1.7 & 192 & 15.3 & 2.2 & 58 & 12.6 & 5 & 14 & 18.6 & 3.7 & 57 & 7.3 & 1.6 & 15 \\
\hline $65-74$ & 13.9 & 1.7 & 100 & 12.6 & 2.5 & 34 & 12.4 & 7.8 & 7 & 19.8 & 3.5 & 24 & 9.3 & 4.1 & 10 \\
\hline $75+$ & 8.7 & 2.2 & 48 & 7.7 & 2.5 & 10 & 14.2 & 6.9 & 5 & 12 & 5.5 & 8 & 10.3 & 4.5 & 6 \\
\hline $\mathrm{F}_{2}^{\mathrm{a}}$ & $22.1^{\star \star \star}$ & & & 2.9 & & & 0 & & & 0.8 & & & 0.3 & & \\
\hline \multicolumn{16}{|l|}{ Gender } \\
\hline Male & 12.5 & 1.4 & 106 & 9.5 & 1.9 & 26 & 19.3 & 10.4 & 11 & 12.5 & 3.3 & 25 & 9.2 & 2.9 & 17 \\
\hline Female & 19.3 & 1.7 & 234 & 15.6 & 1.7 & 76 & 6.1 & 1.6 & 15 & 22.1 & 3.5 & 64 & 8 & 2 & 14 \\
\hline \multirow{2}{*}{\multicolumn{16}{|c|}{ Employment status }} \\
\hline & & & & & & & & & & & & & & & \\
\hline Working & 17.4 & 1.6 & 124 & 11 & 2.3 & 26 & 8.9 & 3.3 & 13 & 17.3 & 6.1 & 26 & 3.3 & 2.5 & 5 \\
\hline Not working & 15.8 & 1.6 & 216 & 14.2 & 1.8 & 76 & 17.9 & 9 & 13 & 18 & 3.2 & 63 & 12 & 2 & 26 \\
\hline $\begin{array}{l}\mathrm{F}_{1} \\
\text { Education }\end{array}$ & 0.7 & & & 1.2 & & & 1 & & & 0 & & & $9.2^{\star \star}$ & & \\
\hline$<$ High school & 16.1 & 2.9 & 66 & 16.1 & 2.5 & 51 & 15.6 & 10.7 & 7 & 18.9 & 2.7 & 56 & 7.9 & 3 & 9 \\
\hline High school & 13.5 & 1.6 & 100 & 12.1 & 2.9 & 29 & 11.2 & 5.7 & 8 & 19.6 & 5.7 & 16 & 13.2 & 4.3 & 9 \\
\hline$>$ High school & 18.8 & 1.5 & 174 & 10.4 & 2.4 & 22 & 10.7 & 5.3 & 11 & 12.8 & 4.9 & 17 & 7.1 & 2.2 & 13 \\
\hline $\begin{array}{l}\mathrm{F}_{2} \\
\text { Poverty-income level }\end{array}$ & \multicolumn{14}{|c|}{ Poverty-income level } & \\
\hline$<100 \%$ poverty & 29.8 & 6.3 & 2.1 & 26.2 & 7.6 & 12 & 13.1 & 8.5 & 3 & 19.6 & 8.1 & 13 & 14.1 & 5.3 & 9 \\
\hline $100-300 \%$ poverty & 15.4 & 2 & 108 & 14.1 & 1.6 & 69 & 15.7 & 8.5 & 13 & 14.8 & 3.1 & 36 & 5.8 & 2.9 & 6 \\
\hline$\geq 300 \%$ poverty & 16 & 1.1 & 211 & 7.5 & 1.6 & 22 & 7.6 & 3.3 & 10 & 20.2 & 4.7 & 40 & 8.1 & 2.3 & 16 \\
\hline $\begin{array}{l}F_{2} \\
\text { Marital status }\end{array}$ & $4.1^{*}$ & & & $5.0^{\star \star}$ & & & 0.6 & & & 0.8 & & & 0.8 & & \\
\hline Married & 14 & 1.4 & 171 & 8.7 & 1.8 & 23 & 12.3 & 6 & 12 & 16.4 & 2.5 & 45 & 9.5 & 2.4 & 27 \\
\hline Not married & 20.2 & 2.4 & 169 & 16.1 & 2.1 & 79 & 13.1 & 5.2 & 14 & 19.9 & 3.7 & 44 & 5.3 & 3.1 & 4 \\
\hline$F_{1}$ & $4.5^{\star}$ & & & $6.1^{*}$ & & & 0 & & & 0.7 & & & 1 & & \\
\hline \multicolumn{16}{|l|}{ Region } \\
\hline Northeast & 17.4 & 3.7 & 75 & 14.5 & 2.8 & 17 & 17.7 & 7.9 & 19 & 15.6 & 5.9 & 20 & 16.4 & 11.9 & 4 \\
\hline Midwest & 15.7 & 2.2 & 97 & 17.6 & 3.6 & 26 & 14.6 & 17.6 & 1 & 8.7 & 5.5 & 3 & 0 & 0 & 0 \\
\hline South & 14.6 & 2 & 98 & 9.7 & 1.4 & 51 & 1.8 & 1.4 & 5 & 21.8 & 2.5 & 52 & 10.1 & 4.8 & 3 \\
\hline West & 18.8 & 2.2 & 70 & 13.3 & 6.6 & 9 & 17.8 & 17.1 & 1 & 16.7 & 4.5 & 14 & 7.3 & 1.2 & 24 \\
\hline $\mathrm{F}_{3}$ & 0.8 & & & 1.9 & & & 1.4 & & & 1.1 & & & $\mathrm{n} / \mathrm{a}$ & & \\
\hline \multicolumn{16}{|l|}{ Nativity } \\
\hline US-born & 16.9 & 1.3 & 333 & 12.5 & 1.4 & 101 & 15.2 & 9.3 & 4 & 17.2 & 3.9 & 14 & 3 & 1.4 & 3 \\
\hline Foreign-born & 5 & 2.2 & 7 & 6.2 & 4.7 & 2 & 10.3 & 3.6 & 22 & 18.2 & 2.6 & 75 & 10 & 2.4 & 28 \\
\hline $\begin{array}{l}F_{1} \\
\text { Among foreign-born only: }\end{array}$ & $11.0^{*}$ & & & 1.3 & & & 0.4 & & & 0 & & & $5.9^{\star}$ & & \\
\hline \multicolumn{16}{|l|}{ Year in the USA } \\
\hline$<10$ years & & & & & & & 43.2 & 15.1 & 4 & 15.9 & 4.8 & 11 & 11.1 & 5 & 8 \\
\hline $11-<20$ years & & & & & & & 11.3 & 6.4 & 6 & 11.7 & 5 & 10 & 7.5 & 3.7 & 7 \\
\hline$\geq 20$ years & & & & & & & 6.7 & 3.1 & 12 & 18.1 & 2.8 & 53 & 10.9 & 3.4 & 13 \\
\hline & \multicolumn{14}{|c|}{ Age at immigration } & \\
\hline$<35$ years & & & & & & & 9.3 & 4.7 & 9 & 16.2 & 3.3 & 37 & 8.1 & 2.9 & 6 \\
\hline$\geq 35$ years & & & & & & & 11 & 4.3 & 12 & 19.2 & 3.9 & 37 & 10.9 & 2.7 & 22 \\
\hline $\mathrm{F}_{1}$ & & & & & & & 0.1 & & & 0.3 & & & 0.8 & & \\
\hline
\end{tabular}

CIDI, Composite International Diagnostic Interview; DSM, Diagnostic and Statistical Manual; CPES, Collaborative Psychiatric Epidemiology Surveys; SE, Standard error.

Any anxiety disorder includes panic disorder, agoraphobia, social phobia, generalized anxiety disorder, and posttraumatic stress disorder.

Prevalence estimates calculated from cross-tabulation of any lifetime anxiety disorder and selected characteristic stratified by race/ethnicity.

a Test of association determined by adjusted Wald $F$-test for each race/ethnicity.

${ }^{*} p<0.05$.

${ }^{* *} p<0.01$

$* * * p<0.001$.

for a disorder than those who are working (3.3\%). Among African Americans, lower income is associated with higher prevalence of both mood and anxiety disorders. Not being married is significantly associated with higher prevalence of mood and anxiety disorders among non-Hispanic White people, higher 
prevalence of anxiety disorders among African Americans, and a higher prevalence of mood disorders among Latinos. White people born in the USA had significantly higher prevalence of both mood and anxiety disorders than those born outside of the USA, whereas foreignborn Asian Americans had a higher prevalence of anxiety disorders than those born in the USA.

\section{Conclusions}

This study examined prevalence rates for psychiatric disorders among a national sample of older nonHispanic White people, African Americans, Caribbean Black people, Latinos, and Asian Americans. Overall, older non-Hispanic White people and Latinos consistently had higher prevalence rates of disorders compared with the other racial and ethnic groups in this study and had significantly greater odds of meeting criteria for a mood or anxiety disorder compared with African Americans. The similarity in prevalence for White people and Latinos is inconsistent with previous research indicating that Latinos, particularly immigrant Latinos, are at lower risk for most psychiatric disorders compared with White people (Alegría et al., 2008; Breslau et al., 2006; Takeuchi et al., 2007). However, recent research has also found that the protective effect of immigration is inconsistent across Latino subgroups and disorders (Alegría et al., 2008; Jimenez et al., 2010; González et al., 2010). Further, the lower risk for disorders among Latinos is more pervasive in younger cohorts and within lower education groups (Breslau et al., 2006). The current study supports Breslau et al.'s finding by indicating that prevalence between White people and Latino older adults is similar. Furthermore, whereas nativity, length of time in the USA, and age of immigration have been postulated as possible reasons for a higher risk of disorders among older Latinos (Burnam et al., 1987; Vega et al., 1998), these factors were not significantly related to prevalence among Latinos in this study. This is consistent with Jimenez et al.'s (2010) findings related to nativity and extends the inquiry by considering additional immigrationrelated variables.

Consistent with prior research, women had higher prevalence for disorders (e.g., Kessler et al., 2005). Additionally, within selected racial/ethnic groups (i.e., White people, African Americans, and Latinos) persons who are not married and not working had higher prevalence for disorders. Current perspectives on the higher prevalence of disorders for persons who are unmarried and unemployed focus on distinguishing between social selection versus social causation processes
(Mastekaasa, 1992). In addition, however, previous research has demonstrated the ways in which the processes of immigration and acculturation can differentially affect men and women (e.g., Black et al., 1998). The higher prevalence of mood disorders among older Latinos who are not working and not married suggests a need to examine the correlates of psychiatric disorders among discrete demographic and ethnic subgroups of the Latino population.

There are several possible explanations for the lower prevalence of major depression and dysthymia among African Americans. Prior evidence suggests that Black people express psychological distress differently than non-Hispanic White people with symptom presentation less focused on mood disturbances and more focused on somatic complaints (Brown et al., 1996; Das et al., 2003). Furthermore, dysphoric mood, suicidal thoughts, agitation, or anxiety may be less characteristic of depression for African Americans (Wohl et al., 1997; Gallo et al., 1998). Moreover, symptoms of depression may be minimized or interpreted by African Americans as part of life's burden to be endured or as normal signs of the aging process (Neal-Barnett and Smith, 1997; Steffens et al., 1997). Thus, the diagnostic measures used in the CPES may not adequately capture symptom presentation, culturally bound explanatory models, and sociocultural experiences. Other plausible explanations for the lower rates of mood disorders among African Americans may be related to increased levels of religious involvement and church-based social support, which are linked to higher levels of psychological well-being (Krause, 2002; Levin et al., 1995). Older African Americans consistently report higher levels of religious involvement (Taylor et al., 2007; Taylor et al., 2004) and informal social support from church members (Krause, 2002). Additionally, research on older African Americans indicates that church attendance was inversely associated with mood disorders (Chatters et al., 2008).

Among African Americans, the higher prevalence of disorders for those with less income and among those who are not married suggest that disorders are associated with fewer material resources and socially supportive relationships and networks. The finding that being married is a protective factor for older African Americans is consistent with previous epidemiological research (Ford et al., 2007). Interestingly, region was not significant in this study although prior research has found lower prevalence of disorders among older Black adult residents of the South who have larger social networks that provide assistance with health and mental health problems (Chatters et al., 1985; Ford et al., 2007). 
Of all the groups studied, Asian Americans were less likely to report any anxiety disorder. This is consistent with recent work based on younger age cohorts, which documented that Asian Americans less frequently endorsed symptoms of all four anxiety disorders compared with other racial groups (Asnaani et al., 2010). Language and cultural differences may influence the degree to which diagnostic instruments capture the meaning of anxiety symptoms among Asian Americans (Alegría et al., 2004; Johnson et al., 2007). Others have also speculated that cultural differences in terms of a collectivistic worldview, emphasizing the importance of maintaining harmonious relationships and overall well-being, may account for lower anxiety rates (Asnaani et al., 2010; Landrine, 1992).

Lastly, immigration-related phenomena such as degree of bilingual fluency, duration of exposure to US culture, and acculturative stress have been suggested as potential explanations for different rates of anxiety among immigrants (Breslau and Chang, 2006; Takeuchi et al., 2007). Jimenez et al. (2010) found that older Asian immigrants had higher lifetime rates of generalized anxiety disorder compared with older Asians born in the USA. Our finding that older foreign-born Asian Americans had a higher prevalence of anxiety disorders than those born in the USA is consistent with this work. However, Asians as a whole consistently have lower rates of most psychiatric disorders compared with other groups in the USA. Further, it is interesting to note that the prevalence of disorders in Asian countries is generally lower than in the USA (WHO World Mental Health Survey Consortium, 2004). Given that immigrants tend to have better health compared with those who remain in the home country, the higher prevalence of anxiety disorders in this sample of foreign-born Asian immigrants lends some support for acculturative stress hypotheses; however, this cannot be directly assessed from these data.

Although prevalence rates of psychiatric disorders differ among racial and ethnic groups, we should avoid the interpretation that some groups have higher versus lower need for mental health services based only on these findings. As alluded to earlier, differential presentation of self-reported psychiatric symptomatology among racial and ethnic groups are a possible explanation for the differences in rates across categories (Alegría and McGuire, 2003; Alegría et al., 2007). Further, other aspects of cultural context may affect symptom presentation of depression among older ethnic and racial minorities beyond the somatization of distress (Diefenbach et al., 2009). This has implications for practice in that clinicians and other service providers will need to consider potential group-specific norms regarding presentation of distress and the meanings of symptoms. Provider factors that may increase clinician bias and misdiagnosis will equally need attention. Although this research could not account for these nuanced approaches to psychiatric diagnosis, the study strongly underscores the need for future research that explores culturally based explanatory models of both symptom presentation and provider interpretation.

\section{Limitations}

This study has several limitations. First, because segments of the population such as homeless and institutionalized individuals were not represented, our findings are not generalizable to these subgroups. Second, disorder-specific symptoms and behaviors may be underreported because of item non-response to sensitive questions, which is a common issue in survey interviewing. Third, we cannot examine discrete groups of Latinos (e.g., Mexican American) or Asian Americans (e.g., Chinese) because of the small sample size of older adults in these groups. Fourth, the relatively small sample sizes of Asian Americans and Caribbean Black people coupled with low prevalence rates makes it more difficult to analyze discrete disorders among these two groups. We did not adjust for health status because this information was not available for non-Hispanic White people. Despite these limitations, this study is one of a recent group of investigations that examines the prevalence rates of psychiatric disorders among five racial and ethnic groups that significantly enhances our understanding of psychiatric disorders within and across older minority groups.

\section{Conflict of interest}

Authors have no conflicts of interest.

Key points

- Major depressive disorder and social phobia were most prevalent among adults aged 55 and older.

- Older non-Hispanic Whites and Latinos high prevalence rates for disorders than other racial and ethnic groups which is inconsistent with previous research.

- Older African Americans had lower rates of depression and dysthymia.

- Asian Americans were less likely than other groups to meet criteria for an anxiety disorder. However, older foreign born Asians had a higher prevalence of anxiety disorders than U.S.-born Asians. 


\section{Acknowledgements}

Funding/Support: Data collection on which this study is based was supported by the National Institute of Mental Health (NIMH; U01-MH57716), the Office of Behavioral and Social Science Research at the National Institutes of Health (NIH), and the University of Michigan. Preparation of this manuscript was supported by grants from the NIMH to KDL, MPA, LMC (R01-MH084963), from NIA to LMC and RJT (R01 AG018782).

\section{References}

Alegría M, McGuire T. 2003. Rethinking a universal framework in the psychiatric symptom-disorder relationship. J Health Soc Behav 44: 257-274. doi: 10.2307/ 1519778

Alegría M, Canino G, Shrout PE, et al. 2008. Prevalence of mental illness in immigrant and non-immigrant U.S. Latino groups. Am J Psychiatry 165: 359-369. doi: 10.1176/appi.ajp.2007.07040704

Alegría M, Shrout PE, Woo M, Guarnaccia P, Sribney W, Vila D, et al. 2007. Understanding differences in past year psychiatric disorders for Latinos living in the US. Social Science and Medicine 65(2): 214-230. doi: 10.1016/j.socscimed.2007.03.026

Alegría M, Takeuchi D, Canino G, et al. 2004. Considering context, place and culture: The National Latino and Asian American Study. Int J Meth Psychiatr Res 13: 208-220. doi: $10.1002 / \mathrm{mpr} .178$

American Association for Public Opinion Research. 2006. Standard Definitions: Final Dispositions of Case Codes and Outcome Rates for Surveys. AAPOR: Lenexa, KS

Asnaani A, Richey A, Dimaite R, Hinton EE, Hofmans SG. 2010. A cross-ethnic comparison of lifetime prevalence rates of anxiety disorders. J Nerv Ment Dis 198: 551-555. doi: 10.1097/NMD.0b013e3181ea169f

Black SA, Markides KS, Miller TQ. 1998. J Gerontol B Psychol Sci Soc Sci 53B: S198-S208.

Brenes GA, Penninx BWJH, Judd PH, et al. 2008. Anxiety, depression, and disability across the lifespan. Aging Ment Health 12: 158-163. doi: 10.1080/13607860601124115

Breslau J, Chang D. 2006. Psychiatric disorders among foreign-born and US-born Asian-Americans in a US national survey. Soc Psychiatry Psychiatr Epidemiol 41: 943-950.

Breslau J, Aguilar-Gaxiola S, Kendler KS, et al. 2006. Specifying race-ethnic differences in risk for psychiatric disorder in a USA national sample. Psychol Med 36: 57-68. doi: 10.1017/S0033291705006161

Brown C, Schulberg HC, Madonia MJ. 1996. Clinical presentations of major depression by African Americans and whites in primary medical care practice. $J$ Affect Disord 41: 181-191. doi: 10.1016/S0165-0327(96)00085-7

Burnam MA, Hough RL, Karno M, Escobar JI, Telles CA. 1987. Acculturation and lifetime prevalence of psychiatric disorders among Mexican Americans in Los Angeles. J Health Soc Behav 28: 89-102. doi: 10.2307/2137143

Callahan CM, Kroenke K, Counsel SR, et al. 2005. Treatment of depression improves physical functioning in older adults. J Am Geriatr Soc 53: 367-373. doi: 10.1111/ j.1532-5415.2005.53151.x

Chatters LM, Bullard KM, Taylor RJ, et al. 2008. Religious participation and DSM-IV disorders among older African Americans: findings from the National Survey of American Life (NSAL). Am J Geriatr Psychiatry 16: 957-965. doi: 10.1097/ JGP.0b013e3181898081

Chatters LM, Taylor RJ, Jackson JS. 1985. Size and composition of the informal helper networks of elderly Blacks. I Gerontol B Psychol Sci Soc Sci 40: 605-614.

Cohen CI, Magai C, Yaffee R, Walcott-Brown L. 2006. The prevalence of anxiety and associated factors in a multiracial sample of older adults. Psychiatr Serv 57 1719-1725. doi: 10.1176/appi.ps.57.12.1719

Das AK, Olfson M, McCurtis HL, Weissman MM. 2003. Depression in African Americans: breaking barriers to detection and treatment. J Fam Pract 55: 30-39.

Diefenbach GJ, Disch WB, Robison JT, Baez E, Coman E. 2009. Anxious depression among Puerto Rican and African-American older adults. Aging Ment Health 13: 118-126. doi: 10.1080/13607860802591062

Ford BC, Bullard KM, Taylor RJ, et al. 2007. Lifetime and twelve-month prevalence of DSM-IV disorders among older African Americans: findings from the National Survey of American Life (NSAL). Am J Geriatr Psychiatry 15: 652-659. doi: 10.1097/JGP.0b013e3180437d9e

Gallo JJ, Cooper-Patrick L, Lesikar S. 1998. Depressive symptoms of whites and African Americans aged 60 years and older. J Gerontol B Psychol Sci Soc Sci 53: 277-286.
González HM, Tarraf W, Whitfield KE, Vega WA. 2010. The epidemiology of major depression and ethnicity in the United States. J Psychiatr Res 44: 1043-1051. doi: 10.1016/j.jpsychires.2010.03.017

Grant BF, Hasin DS, Stinson FS, et al. 2005. Prevalence, correlates, co-morbidity, and comparative disability of DSM-IV generalized anxiety disorder in the USA: Results from the National Epidemiologic Survey on Alcohol and Related Conditions. Psychol Med 35: 1747-1759. doi: 10.1017/S0033291705006069

Hasin DS, Goodwin RD, Stinson FS, Grant BF. 2005. Epidemiology of major depressive disorder: results from the National Epidemiologic Survey on Alcoholism and Related Conditions. Arch Gen Psychiatry 62: 1097-1106. doi: 10.1001/archpsyc. 62.10 .1097

Heeringa SG, Berglund, P. 2007. National Institutes of Mental Health (NIMH) Collaborative Psychiatric Epidemiology Survey Program (CPES) Data Set: Integrated Weights and Sampling Error Codes for Design-Based Analysis. University of Michigan Survey Research Center: Ann Arbor.

Heeringa SG, Wagner J, Torres M, et al. 2004. Sample designs and sampling methods for the Collaborative Psychiatric Epidemiology Studies (CPES). Int J Meth Psychiatr Res 13: 221-240. doi: 10.1002/mpr.179

Jimenez DE, Alegría M, Chen C, Chan D, Laderman M. 2010. Prevalence of psychiatric illnesses in older ethnic minority adults. J Am Geriatr Soc 58: 256-264. doi: $10.1111 / j .1532-5415.2009 .02685 . x$

Johnson MR, Hartzema AG, Mills TL, et al. 2007. Ethnic differences in the reliability and validity of a panic disorder screen. Ethnic Health 12: 283-296. doi: 10.1080/ 13557850701235069

Kessler RC, Üstün TB. 2004. The World Mental Health (WMH) survey initiative version of the World Health Organization (WHO) Composite International Diagnostic Interview (CIDI). Int J Meth Psychiatr Res 13: 93-121. doi: 10.1002/mpr.168

Kessler RC, Berglund P, Demler O, Jin R, Walters EE. 2005. Lifetime prevalence and age-of-onset distributions of DSM-IV disorders in the National Comorbidity Survey Replication. Arch Gen Psychiatry 62: 593-602. doi: 10.1001/ archpsyc.62.6.593

Krause N. 2002. Church-based social support and health in old age: exploring variations by race. J Gerontol B Psychol Sci Soc Sci 57: S332-S347.

Landrine H. 1992. Clinical implications of cultural differences: the referential versus the indexical self. Clin Psychol Rev 12: 401-415. doi: 10.1016/0272-7358(92) 90124-Q

Levin JS, Chatters LM, Taylor RJ. 1995. Religious effects on health status and life satisfaction among Black Americans. J Gerontol B Psychol Sci Soc Sci 50: S154-S163.

Lyness JM, Niculescu A, Tu X, Reynolds CF, Caine ED. 2006. The relationship of medical comorbidity and depression in older, primary care patients. Psychosomatics 47: 435-439. doi: 10.1176/appi.psy.47.5.435

Mastekaasa A. 1992. Marriage and psychological well-being: some evidence on selection into marriage. Journal of Marriage and Family 54: 901-911

Mehta KM, Simonsick EM, Penninx B, et al. 2003. Prevalence and correlates of anxiety symptoms in well-functioning older adults: findings from the Healthy Aging and Body Composition Study. J Am Geriatr Soc 51: 499-504. doi: 10.1046/ j.1532-5415.2003.51158.x

Mojtabai R, Olfson M. 2004. Cognitive deficits and the course of major depression in a cohort of middle-aged and older community-dwelling adults. J Am Geriatr Soc 52: 1060-1069. doi: 10.1111/j.1532-5415.2004.52302.x

Neal-Barnett AM, Smith J. 1997. African Americans. In Cultural Issues in the Treatment of Anxiety, Friedman S (ed.). The Guilford Press: New York.

Ortega AN, Rosenheck RA, Alegría M, Desai RA. 2000. Acculturation and the lifetime risk of psychiatric and substance use disorders among Hispanics. J Nerv Ment Dis 188: 728-735. doi: 10.1097/00005053-200011000-00002

Pennell B-E, Bowers A, Carr D, et al. 2004. The development and implementation of the National Comorbidity Survey Replication, the National Survey of American Life, and the National Latino and Asian American Survey. Int J Meth Psychiatr Res 13: 241-269. doi: 10.1002/mpr.180

Ramsawh HJ, Raffa SD, Edelen MO, Rende R, Keller MB. 2009. Anxiety in middle adulthood: effects of age and time on the 14-year course of panic disorder, social phobia and generalized anxiety disorder. Psychol Med 39: 615-624. doi: 10.1017/ S0033291708003954

Scott KM, Von Korff M, Alonso J, et al. 2008. Age patterns in the prevalence of DSMIV depressive/anxiety disorders with and without physical co-morbidity. Psychol Med 38: 1659-1669. doi: 10.1017/S0033291708003413

Smith SM, Stinson FS, Dawson DA, et al. 2006. Race/ethnic differences in the prevalence and co-occurrence of substance use disorders and independent mood and anxiety disorders: results from the National Epidemiologic Survey on Alcohol and Related Conditions. Psychol Med 36: 987-998.

Steffens DC, Artigues DL, Ornstein KA, Krishnan KRR. 1997. A review of racial differences in geriatric depression: implications for care and clinical research. J Natl Med Assoc 89: 731-736.

Takeuchi D, Alegría M, Jackson JS, Williams DR. 2007. Immigration and mental health: diverse findings in Asian, Black, and Latino populations. Am J Public Health 97: 11-12. doi: 10.2105/AJPH.2006.103911

Taylor RJ, Chatters LM, Jackson JS. 2007. Religious and spiritual involvement among older African Americans, Caribbean blacks, and non-Hispanic whites. J Gerontol B Psychol Sci Soc Sci 62B: S238-S250. 
Taylor RJ, Chatters LM, Levin JS. 2004. Religion in the Lives of African Americans: Social, Psychological and Health Perspectives. Sage: Newbury Park.

Teachman BA. 2006. Aging and negative affect: the rise and fall and rise of anxiety and depression symptoms. Psychol Aging 21: 201-207. doi: 10.1037/0882-7974.21.1.201

Vega WA, Bohdan K, Aguilar-Gaxiola S, et al. 1998. Lifetime prevalence of DSM-III-R psychiatric disorders among urban and rural Mexican Americans in California. Arch Gen Psychiatry 55: 771-778. doi: 10.1001/archpsyc.55.9.771
WHO World Mental Health Survey Consortium. 2004. The prevalence, severity, and unmet need for treatment of mental disorders in the World Health Organization World Mental Health Surveys. J Am Med Assoc 291: 2581-2590.

Wohl M, Lesser I, Smith M. 1997. Clinical presentations of depression in African American and White outpatients. Cult Divers Ment Health 3: 279-284. doi: 10.1037/1099-9809.3.4.279 